\title{
The Cost of Prolonged Hospitalization due to Postthyroidectomy Hypocalcemia: A Case-Control Study
}

\author{
Navid Zahedi Niaki, ${ }^{1}$ Harmeet Singh, ${ }^{1}$ Sami P. Moubayed, ${ }^{1}$ Rebecca Leboeuf, ${ }^{2}$ \\ Jean-Claude Tabet, ${ }^{1}$ Apostolos Christopoulos, ${ }^{1}$ Tareck Ayad, ${ }^{1}$ Marie-Jo Olivier, ${ }^{1}$ \\ Louis Guertin, ${ }^{1}$ and Eric Bissada ${ }^{1}$ \\ ${ }^{1}$ Otolaryngology-Head and Neck Surgery Service, Department of Surgery, Université de Montréal Hospital Center (CHUM), \\ 1560 Sherbrooke E, Montreal, QC, Canada H2L 4M1 \\ ${ }^{2}$ Endocrinology Service, Department of Medicine, CHUM, Montreal, QC, Canada H2L 4M1 \\ Correspondence should be addressed to Eric Bissada; ericbissada@yahoo.com
}

Received 17 September 2014; Revised 11 December 2014; Accepted 11 December 2014; Published 28 December 2014

Academic Editor: Pere Berbel

Copyright (C) 2014 Navid Zahedi Niaki et al. This is an open access article distributed under the Creative Commons Attribution License, which permits unrestricted use, distribution, and reproduction in any medium, provided the original work is properly cited.

\begin{abstract}
The aim of this study is to evaluate the additional costs associated with calcium monitoring and treatment as well as evaluate the incidence and predictors of postthyroidectomy hypocalcemia. Methods. This case-control study involved thyroidectomy and completion thyroidectomy patients operated on between January 2012 and August 2013. Cases were defined as requiring calcitriol supplementation, and controls did not require supplementation. Patient (age, sex), nodule (cytology, pathology), surgical data (neck dissection, parathyroid identification, and reimplantation), and hospital stay (days hospitalized in total and after drain removal) were compared. Comparisons were made using $t$-tests and chi-square tests with an alpha of 0.05 . The estimated cost associated with the extended stay was then compared with the cost of supplementation. Results. A total of 191 patients were evaluated (61 cases and 130 controls). Predictors of hypocalcemia include female age, neck dissection, and parathyroid reimplantation. Hypocalcemic patients were hospitalized for a longer period of time after drain removal (2.5 versus 0.8 days, $P<0.001)$, and hospitalization costs after neck drain removal were higher in this group as well $(8,367.32 \$$ versus $2,534.32 \$, P<0.001)$. Conclusion. Postoperative hypocalcemia incurs significant additional health care costs at both the local and health care system levels.
\end{abstract}

\section{Introduction}

As thyroid cancer has the most rapidly rising incidence rate of all major cancers in Canada [1], surgeons will likely be performing an increasing number of thyroidectomies in the foreseeable future. One of the major complications following completion and total thyroidectomy is postoperative hypocalcemia [2], which is mainly due to hypoparathyroidism when the parathyroid glands are put at risk of devascularization, with incidence rates of up to $33 \%$ being reported in centers where it is managed only on the basis of clinical observation and calcium monitoring [3].

Although some research has focused on developing protocols and guidelines to reduce the incidence of postoperative hypocalcemia [4-6], there is very little data on the economic impact of the monitoring and treatment of postthyroidectomy hypocalcemia in Canada.

The current standard of practice at our tertiary hospital center is of postoperative serum calcium monitoring and treatment of hypocalcemia when necessary. The aim of this study is to evaluate the incidence and the predictors of postoperative hypocalcemia following thyroid surgery as well as to evaluate the additional cost of prolonged hospitalization incurred by the surveillance and treatment of hypocalcemia.

\section{Methods}

We have conducted a retrospective cohort study on all patients undergoing total or completion thyroidectomy at our tertiary referral hospital center between January 2012 and 
August 2013. Excluded patients were those receiving preoperative calcium and/or vitamin $\mathrm{D}$ supplementation and patients with chronic kidney disease, hyperparathyroidism, reexploration for recurrent goiter, and previous parathyroidectomy. Patients whose hospitalization had been prolonged from complications other than hypocalcemia (i.e., chyle leak) were also excluded. A flow diagram is presented in Figure 1.

The standard protocol for total thyroidectomy at our center involves perioperative identification of both recurrent laryngeal nerves and surgical drain installation in all patients. Discharge from our department is granted upon drainage less than $20 \mathrm{cc}$ in 24 hours. The postthyroidectomy hypocalcemia protocol at our center requires monitoring for Chvostek and Trousseau signs every 8 hours and the value of ionized $\mathrm{Ca}^{2+}$ every 8 hours. If ionized $\mathrm{Ca}^{2+}$ values are less than $1 \mathrm{mmol} / \mathrm{L}$, a calcium gluconate bolus is administered followed by a perfusion which is decreased when ionized $\mathrm{Ca}^{2+}$ levels reach at least 1.11. If ionized $\mathrm{Ca}^{2+}$ falls below $1 \mathrm{mmol} / \mathrm{L}$, oral calcium (1 g t.i.d.) and calcitriol ( 0.25 mcg b.i.d.) are also administered with the perfusion.

We have collected patient (sex, age), nodule (cytology, pathology), and surgical (neck dissection, parathyroids identified, and parathyroid reimplantation) data.

The two main outcomes are the incidence of hypocalcemia and the number of days hospitalized. Hypocalcemia was defined by the need for calcitriol supplementation at hospital discharge. Permanent hypocalcemia was defined as hypocalcemia extending beyond six months postoperatively. The number of days hospitalized was evaluated as the total number of days and the number of days hospitalized following neck drain removal, which represents the added time required for monitoring and/or treatment of hypocalcemia.

Patients were divided into two groups: normocalcemic and hypocalcemic. Patient, nodule, and surgical data were compared between the two groups to identify predictors of hypocalcemia. Hospitalization following neck drain removal represents the additional time required for calcium monitoring in the normocalcemic group and the additional time required for hypocalcemia treatment in the hypocalcemic group. Hospitalization data was compared between groups to evaluate the additional hospitalization time incurred by hypocalcemia treatment. Total hospitalization cost was estimated based on an average hospitalization cost of 3336\$/day on the ward. This value was obtained from our admissions department and only accounts for basic care giving expenses and blood tests on wards and excludes the intensive care unit.

Categorical variables were compared between groups using the chi-square, and hospitalization days and costs were compared using Student's $t$-test. All analyses were conducted using SPSS 17.0 (SPSS, Chicago, IL) with a $P$ less than 0.05 considered statistically significant.

\section{Results}

A total of 191 patients were included. Patient and nodule details are presented in Table 1 . Of the 191 patients that underwent surgery, 31.9\% (61 cases) developed hypocalcemia and $68.1 \%$ (130 controls) were normocalcemic. The incidence
TABLE 1: Patients and nodule details.

\begin{tabular}{lccc}
\hline & $\begin{array}{c}\text { Normocalcemic } \\
\text { group }(n=130)\end{array}$ & $\begin{array}{c}\text { Hypocalcemic } \\
\text { group }(n=61)\end{array}$ & $P$ value \\
\hline Sex & & & \\
$\quad$ Male & $41(31.5 \%)$ & $10(16.4 \%)$ & 0.027 \\
$\quad$ Female & $89(68.5 \%)$ & $51(83.6 \%)$ & \\
Mean age (SD) & $48.0(13.3)$ & $46.2(15.4)$ & 0.412 \\
Cytology & & & \\
$\quad$ Benign & $56(43.1 \%)$ & $21(34.4 \%)$ & 0.162 \\
$\quad$ Malignant & $55(42.3 \%)$ & $33(54.1 \%)$ & \\
Pathology & & $11(18.0 \%)$ & 0.310 \\
$\quad$ Benign & $32(24.6 \%)$ & $50(82.0 \%)$ & \\
$\quad$ Malignant & $98(75.4 \%)$ & & \\
\hline
\end{tabular}

TABLE 2: Operative details.

\begin{tabular}{lccc}
\hline & $\begin{array}{c}\text { Normocalcemic } \\
\text { group }(n=130)\end{array}$ & $\begin{array}{c}\text { Hypocalcemic } \\
\text { group }(n=61)\end{array}$ & $P$ value \\
\hline $\begin{array}{l}\text { Surgery type } \\
\quad \text { Completion }\end{array}$ & $21(16.1 \%)$ & $6(9.8 \%)$ & 0.243 \\
$\quad \begin{array}{l}\text { Total } \\
\text { Neck dissection }\end{array}$ & $109(83.8 \%)$ & $55(90.2 \%)$ & \\
$\quad$ No neck dissection & $112(86.2 \%)$ & $42(68.9 \%)$ & 0.005 \\
$\quad$ Neck dissection & $18(13.8 \%)$ & $19(31.1 \%)$ & \\
$\begin{array}{l}\text { Parathyroid } \\
\text { reimplantation }\end{array}$ & & & \\
$\quad \begin{array}{l}\text { No reimplantation } \\
\text { Reimplantation }\end{array}$ & $115(88.5 \%)$ & $44(72.1 \%)$ & 0.005 \\
$\begin{array}{l}\text { Mean number of } \\
\text { parathyroids } \\
\text { identified (SD) }\end{array}$ & $15(11.5 \%)$ & $17(27.9 \%)$ & \\
\hline
\end{tabular}

of postoperative hypocalcemia was $36.4 \%$ (51/140) in women and $24.4 \%(10 / 41)$ in men $(P<0.27)$. There were no statistically significant differences in mean age, cytology, or pathology between the two groups.

Operative characteristics are presented in Table 2. The operative characteristics show a significantly higher percentage of neck dissections and parathyroid reimplantation in the hypocalcemic group. The number of parathyroids identified was similar between both groups with an average of a little over two (2.3 and 2.1, resp., in the normocalcemic and hypocalcemic group). As for the type of surgery, total thyroidectomies were slightly more prevalent in the hypocalcemic group.

Hospitalization details are presented in Table 3. The hospitalization characteristics show statistically longer total number of days hospitalized (5.7 versus 3.1 ) and number of days hospitalized after drain removal (2.5 versus 0.8 ) in the hypocalcemic patients. Hospitalization following neck drain removal represents the additional time needed to monitor the calcium in the normocalcemic group and the additional time required for hypocalcemia treatment in the hypocalcemic group. 


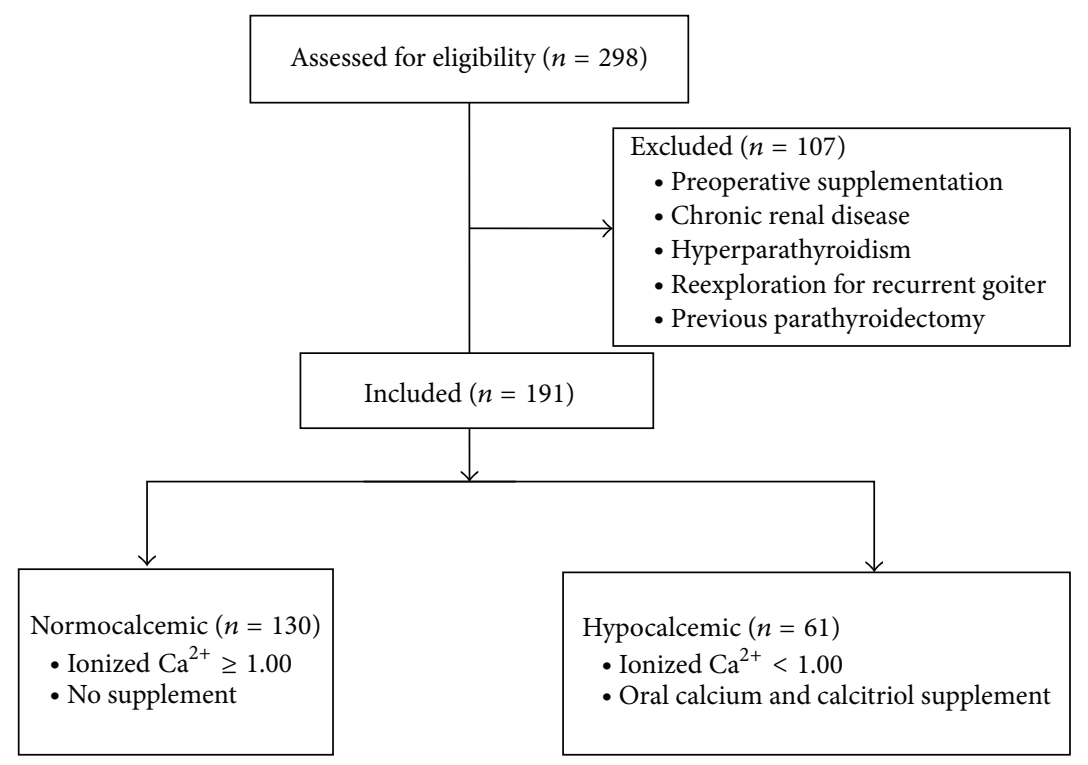

FIgURE 1: Flow diagram of study.

TABLE 3: Hospitalization details.

\begin{tabular}{|c|c|c|c|}
\hline & $\begin{array}{l}\text { Normocalcemic } \\
\text { group }(n=130)\end{array}$ & $\begin{array}{l}\text { Hypocalcemic } \\
\text { group }(n=61)\end{array}$ & $P$ \\
\hline $\begin{array}{l}\text { Total number of } \\
\text { days hospitalized } \\
\text { (SD) }\end{array}$ & $3.1(1.0)$ & $5.7(3.6)$ & $<0.001$ \\
\hline $\begin{array}{l}\text { Average cost of } \\
\text { total number of } \\
\text { days hospitalized } \\
(\mathrm{SD})^{*}\end{array}$ & $\begin{array}{l}10,495.57 \$ \\
(3,299.90 \$)\end{array}$ & $\begin{array}{c}18,976.92 \$ \\
(12,128.57 \$)\end{array}$ & $<0.001$ \\
\hline $\begin{array}{l}\text { Number of days } \\
\text { hospitalized after } \\
\text { drain removal (SD) }\end{array}$ & $0.8(1.1)$ & $2.5(3.4)$ & $<0.001$ \\
\hline $\begin{array}{l}\text { Average cost of } \\
\text { days hospitalized } \\
\text { after drain removal } \\
(\mathrm{SD})^{*}\end{array}$ & $\begin{array}{c}2,534.32 \$ \\
(3,677.53 \$)\end{array}$ & $\begin{array}{c}8,367.32 \$ \\
(11,339.50 \$)\end{array}$ & $<0.001$ \\
\hline
\end{tabular}

${ }^{*}$ Based on the average cost of one day of hospitalization on the ward.

\section{Discussion}

Our findings show that predictors of postthyroidectomy hypocalcemia include female age, neck dissection, and parathyroid reimplantation. Hypocalcemic patients were hospitalized for a longer period of time after drain removal (2.5 versus 0.8 days, $P<0.001$ ), and hospitalization costs per patient after neck drain removal were higher in this group as well $(8,367.35 \$$ versus $2,534.33 \$, P<0.001)$. These additional costs, for both groups, are due to the monitoring and treatment of hypocalcemia, as we excluded other causes of prolonged hospitalization.

The incidence of transient and permanent hypocalcemia in our study is similar to what has been reported in the literature. Studies report transient hypocalcemia rates ranging from $7 \%$ to $25 \%$ and permanent from $0.4 \%$ to $13.8 \%$ [7].
We have identified the same predictors of postthyroidectomy hypocalcemia as in the literature [8]. There is a higher percentage of patients who had neck dissections $(31.1 \%$ versus $13.8 \%)$ and parathyroid reimplementation (27.9\% versus $11.5 \%$ ) in the hypocalcemic group and that difference is statistically significant. We have also found a higher proportion of women becoming hypocalcemic, which is well documented in the literature [9]. Our results do not show the type of surgery as one of the predictors, although there is a trend towards a higher percentage of total thyroidectomy in the hypocalcemic group. With a larger number of patients, that difference could have been statistically significant. Lastly, the high incidence of primary thyroid cancer in our cohort (148 malignant versus 43 benign) follows a trend seen in other recent and similarly designed studies $[10,11]$.

We have successfully evaluated the incidence and the costs of monitoring and treatment of postthyroidectomy hypocalcemia in Canada. The main limitation of our study is that, to evaluate the additional costs incurred by the monitoring and treatment of hypocalcemia, we used an estimate based on the average cost of one day of hospitalization on the ward. The numbers provided to us by the hospital administration are those on the ward and are not pertaining to the intensive care unit. This estimate is accurate as the days of hospitalization after drain are only due to calcium monitoring and management, because we excluded all other causes of prolonged hospitalization. However, the added costs of missed work days, additional hospital visits, outpatient medication, and outpatient laboratory testing due to hypocalcemia were not accounted for and should be included should other investigators wish to evaluate all economic costs of hypocalcemia after thyroidectomy.

The importance of our study resides in showing that the monitoring and treatment of postthyroidectomy hypocalcemia incur significant health care costs. Some studies [3, $4,6]$ have shown that supplementation protocols containing 
calcium with or without calcitriol can prevent the hypocalcemia that occurs after a thyroid surgery. The first type of protocol is routine supplementation in which every patient is supplemented with progressive withdrawal when there are no symptoms of hypocalcemia [6]. Calcium supplementation is given to all patients following thyroidectomy with the addition of calcitriol for high-risk individuals. Second, selective protocols have been described where patients at high risk of hypoparathyroidism are prophylactically supplemented $[5,6,12]$. These protocols rely on the evaluation of preoperative, intraoperative, and/or postoperative PTH levels and require frequent blood sampling. Several PTH levels have been described for prophylactic supplementation: difference of $70 \%$ between pre- and 1-hour postoperative values, absolute intraoperative value, absolute 1-hour postoperative value, and absolute 4-hour postoperative value. A recent cost-utility analysis study has shown that selective protocols would cost slightly more than a routine protocol mainly due to the cost of frequent blood testing [12].

\section{Conclusion}

We have shown that the monitoring and treatment of hypocalcemia incur significant health care costs at the hospital level, especially for the patients that become hypocalcemic. Hypocalcemic patients were hospitalized for a longer period of time after drain removal ( 2.5 versus 0.8 days, $P<0.001$ ), and hospitalization costs after neck drain removal were higher in this group as well $(8,367.35 \$$ versus $2,534.33 \$, P<$ 0.001 ). Strategies to avoid prolonged hospital stays due to hypocalcemia must be instated. Future directions include the identification of the optimal protocol to reduce health care costs pertaining to postthyroidectomy hypocalcemia in the setting of limited resources in the health care system.

\section{Conflict of Interests}

The authors declare that there is no conflict of interests regarding the publication of this paper.

\section{References}

[1] Canadian Cancer Society's Advisory Committee on Cancer Statistics, "Canadian Cancer Statistics 2013 Special topic: Liver cancer. [S.1.]," Canadian Cancer Society; 2013, http://myaccess library.utoronto.ca/login?url=http://site.ebrary.com/lib/utoronto/Top?id=10746792.

[2] L. Rosato, N. Avenia, P. Bernante et al., "Complications of thyroid surgery: analysis of a multicentric study on 14,934 patients operated on in Italy over 5 years," World Journal of Surgery, vol. 28, no. 3, pp. 271-276, 2004.

[3] V. Cote, N. Sands, M. P. Hier et al., "Cost savings associated with post-thyroidectomy parathyroid hormone levels," Otolaryngology-Head and Neck Surgery, vol. 138, no. 2, pp. 204-208, 2008.

[4] D. Glinoer, G. Andry, G. Chantrain, and N. Samil, "Clinical aspects of early and late hypocalcaemia after thyroid surgery," European Journal of Surgical Oncology, vol. 26, no. 6, pp. 571$577,2000$.
[5] R. J. Payne, M. A. Tewfik, M. P. Hier et al., "Benefits resulting from 1- and 6-hour parathyroid hormone and calcium levels after thyroidectomy," Otolaryngology-Head and Neck Surgery, vol. 133, no. 3, pp. 386-390, 2005.

[6] A. Alhefdhi, H. Mazeh, and H. Chen, "Role of postoperative vitamindand/or calcium routine supplementation in preventing hypocalcemia after thyroidectomy: asystematic review and meta-analysis," The Oncologist, vol. 18, no. 5, pp. 533-542, 2013.

[7] P. W. Flint and C. W. Cummings, Cummings OtolaryngologyHead \& Neck Surgery, Mosby/Elsevier, Philadelphia, Pa, USA, 5th edition, 2010.

[8] B. Abboud, G. Sleilaty, C. Braidy et al., "Careful examination of thyroid specimen intraoperatively to reduce incidence of inadvertent parathyroidectomy during thyroid surgery," Archives of Otolaryngology: Head and Neck Surgery, vol. 133, no. 11, pp. 11051110, 2007.

[9] N. B. Sands, R. J. Payne, V. Côté, M. P. Hier, M. J. Black, and M. Tamilia, "Female gender as a risk factor for transient postthyroidectomy hypocalcemia," Otolaryngology-Head and Neck Surgery, vol. 145, no. 4, pp. 561-564, 2011.

[10] A. AlQahtani, A. Parsyan, R. Payne, and R. Tabah, "Parathyroid hormone levels 1 hour after thyroidectomy: an early predictor of postoperative hypocalcemia," Canadian Journal of Surgery, vol. 57, no. 4, pp. 237-240, 2014.

[11] T. N. Le, P. D. Kerr, D. E. Sutherland, and P. Lambert, "Validation of 1-hour post-thyroidectomy parathyroid hormone level in predicting hypocalcemia," Journal of Otolaryngology-Head and Neck Surgery, vol. 43, article 5, 2014.

[12] T. S. Wang, K. Cheung, S. A. Roman, and J. A. Sosa, “To supplement or not to supplement: a cost-utility analysis of calcium and vitamin D repletion in patients after thyroidectomy," Annals of Surgical Oncology, vol. 18, no. 5, pp. 1293-1299, 2011. 


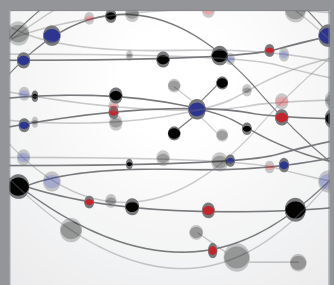

The Scientific World Journal
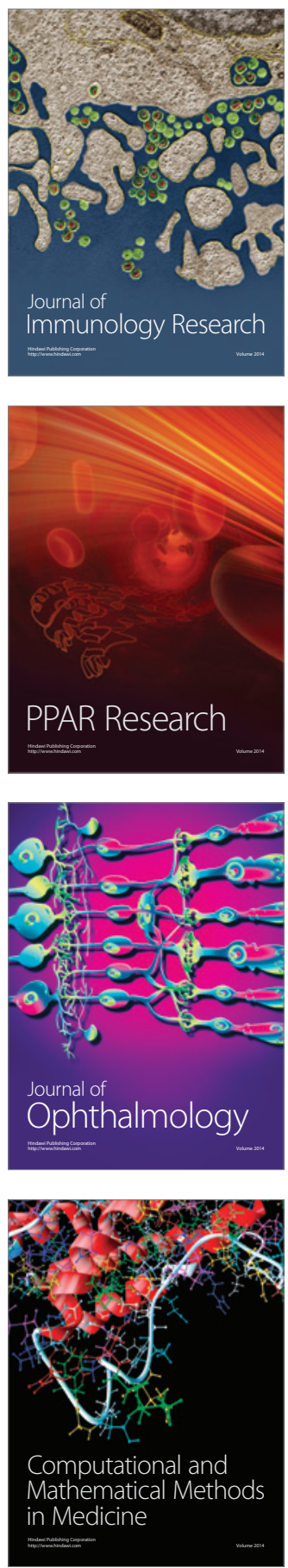

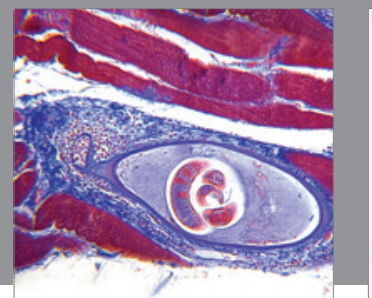

Gastroenterology

Research and Practice
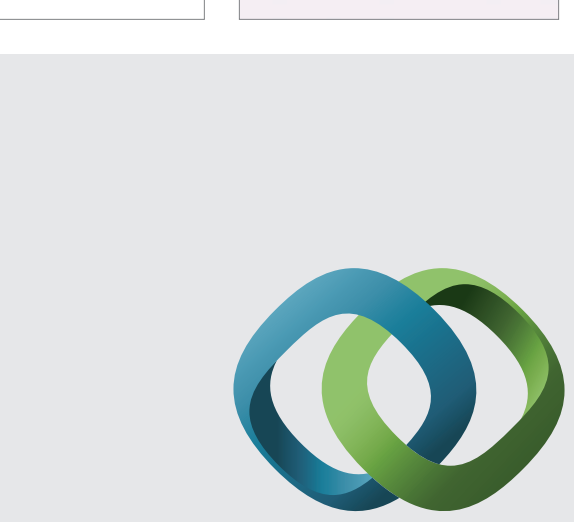

\section{Hindawi}

Submit your manuscripts at

http://www.hindawi.com
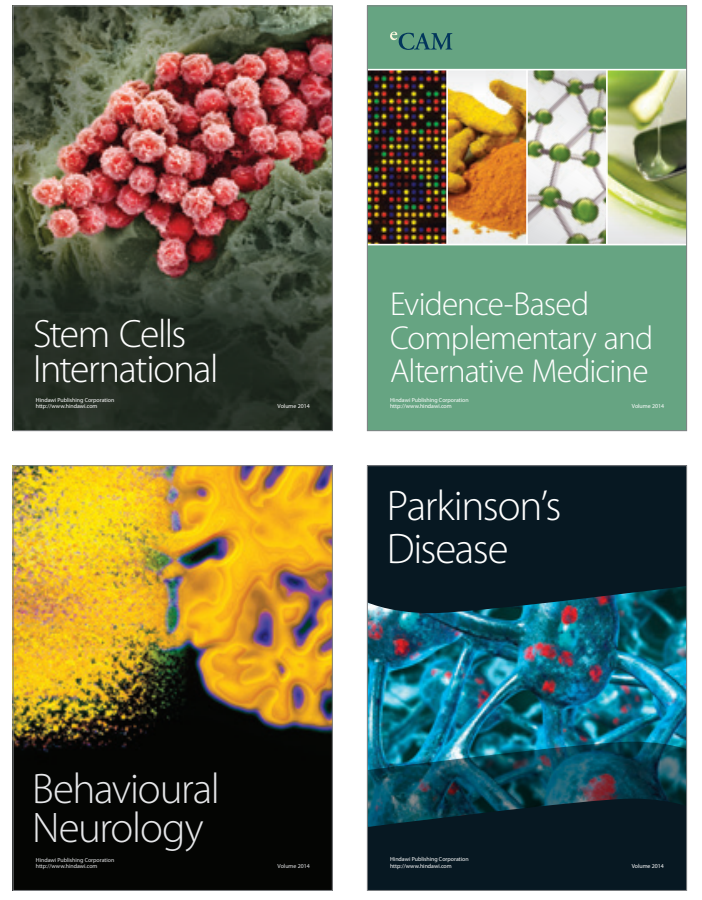
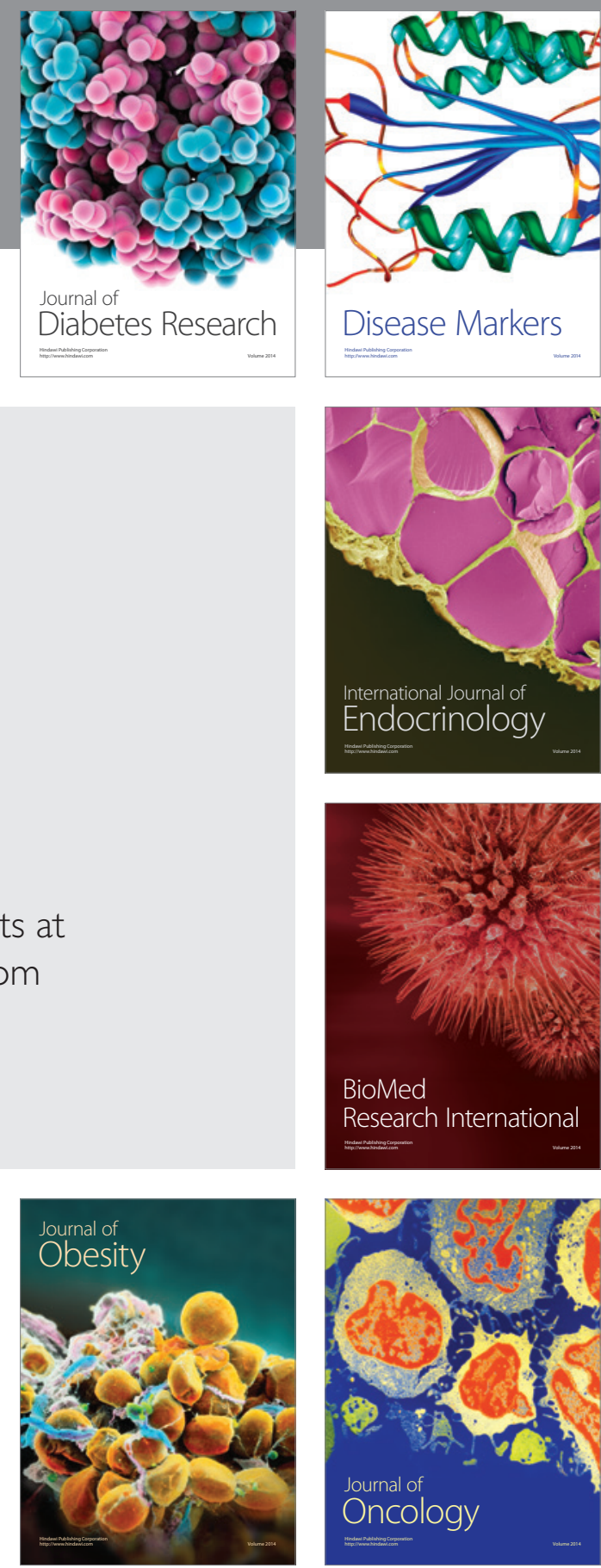

Disease Markers
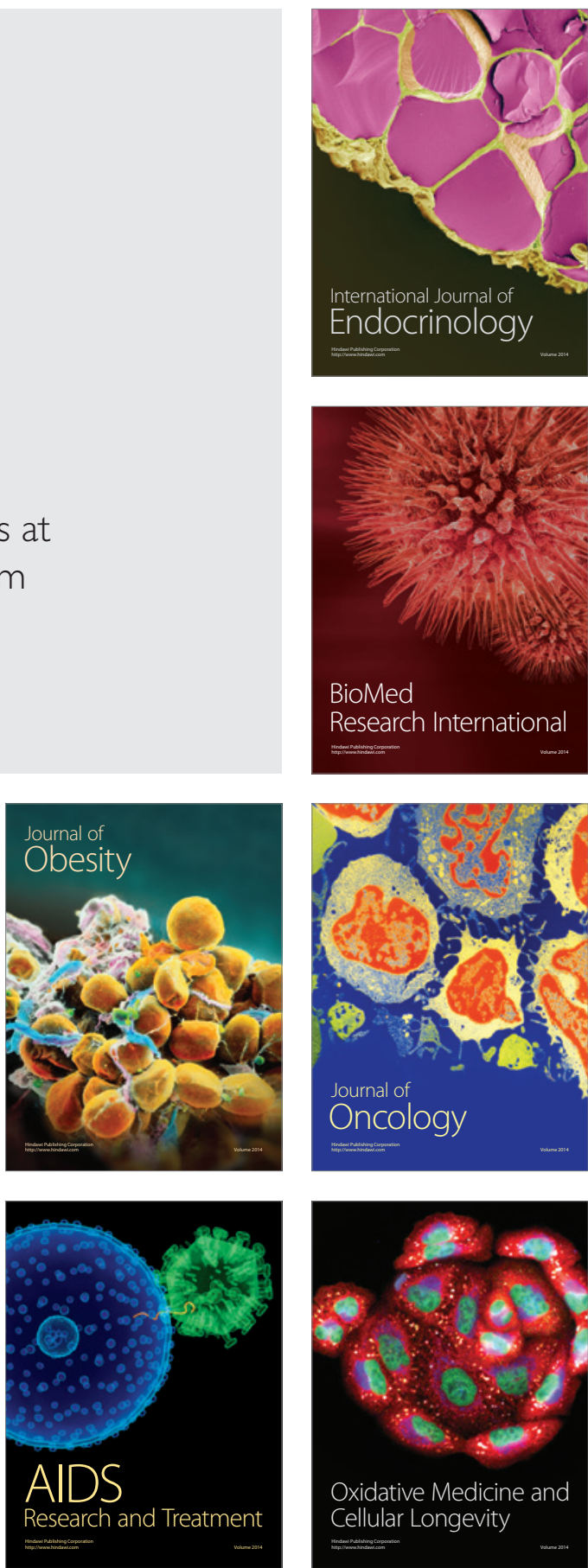